\begin{tabular}{|c|c|c|c|c|c|c|}
\hline \multirow{4}{*}{ Impact Factor: } & ISRA (India) & $=3.117$ & SIS (USA) & $=0.912$ & ICV (Poland) & $=6.630$ \\
\hline & ISI (Dubai, UAE & $=\mathbf{0 . 8 2 9}$ & РИНЦ (Russia & $=0.156$ & PIF (India) & $=1.940$ \\
\hline & GIF (Australia) & $=0.564$ & ESJI (KZ) & $=8.716$ & IBI (India) & $=4.260$ \\
\hline & JIF & $=1.500$ & SJIF (Morocco & $=5.667$ & OAJI (USA) & $=0.350$ \\
\hline
\end{tabular}

SOI: $\underline{1.1 / \mathrm{TAS}} \quad$ DOI: $\underline{10.15863 / \mathrm{TAS}}$
International Scientific Journal
Theoretical \& Applied Science
$\begin{array}{ll}\text { p-ISSN: } 2308-4944 \text { (print) } \quad \text { e-ISSN: } 2409-0085 \text { (online) } \\ \text { Year: } 2019 \quad \text { Issue: } 03 \quad \text { volume: } 71 \\ \text { Published: } 11.03 .2019 \quad \underline{\text { http://T-Science.org }}\end{array}$

SECTION 4. Computer science, computer engineering and automation.
QR - Issue

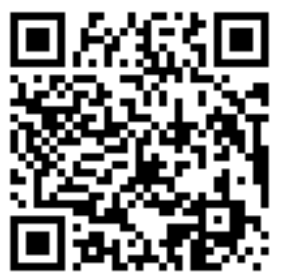

QR - Article

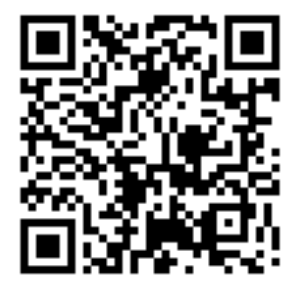

Vadim Andreevich Kozhevnikov

Senior Lecturer,

Peter the Great St.Petersburg Polytechnic University vadim.kozhevnikov@gmail.com

Yulia Efimovna Shats Student

Peter the Great St.Petersburg Polytechnic University jeshats@gmail.com

\title{
RESEARCH OF MEDICAL DATA EXCHANGE STANDARDS
}

Abstract: This article examines some of the existing medical standards and creates a comparative table reflecting the advantages and disadvantages of each.

Key words: HL7 v2, HL7 v3, terminology services, HL7 FHIR.

Language: Russian

Citation: Kozhevnikov, V. A., \& Shats, Y. E. (2019). Research of Medical Data Exchange Standards. ISJ Theoretical \& Applied Science, 03 (71), 62-65.

Soi: http://s-o-i.org/1.1/TAS-03-71-8 Doi: crossef https://dx.doi.org/10.15863/TAS.2019.03.71.8

\section{ИССЛЕДОВАНИЕ СТАНДАРТОВ ОБМЕНА МЕДИЦИНСКИМИ ДАННЫМИ}

Аннотация: В данной статье проводится исследование некоторых существующих медищинских стандартов и создается сравнительная таблица, отражающая преимущества и недостатки каждого из нux.

Ключевые слова: HL7 v2, HL7 v3, стандарты обмена данными, HL7 FHIR.

\section{1 Введение}

Современные информационные системы здравоохранения должны собирать клинические данные в структурированном и, предпочтительно, кодированном формате. Это имеет решающее значение для обмена данными между информационными системами здравоохранения, анализом, качеством и исследованиями медицинских данных, системами поддержки принятия клинических решений, административными функциями и т. д. Но, существует проблема, связанная с тем, что естественный язык огромен и очень богат деталями, но в то же время неоднозначен; он имеет большую зависимость от контекста и использует жаргон и аббревиатуры [1]. Невзирая на то, что был достигнут значительный прогресс в методах обработки знаний и естественного языка, результат пока не является достаточным для использования свободного текста в клинической документации. Для решения данной проблемы были разработаны многочисленные терминологические системы для систематической регистрации клинических данных. Эти системы связывают понятия определенной области и предоставляют ссылку на связанные термины, возможные определения и коды. В целях решения компромисса между захватом данных со свободным текстом и в то же время данными кодирования для компьютерной обработки, в различные периоды времени были разработаны многочисленные терминологические системы для систематической регистрации клинических данных. Статья призвана исследовать формирование некоторых существующих стандартов обмена медицинскими данными и определить наиболее актуальный на сегодняшний день.

\section{2 Современные стандарты}

В связи с тем, что создание единой системы для обмена информацией, все еще является недостижимой целью до сих пор, существует 


\begin{tabular}{|c|c|c|c|c|c|c|}
\hline \multirow{4}{*}{ Impact Factor: } & ISRA (India) & $=3.117$ & SIS (USA) & $=0.912$ & ICV (Poland) & $=6.630$ \\
\hline & ISI (Dubai, UAE & $=0.829$ & РИНЦ (Russia & $=0.156$ & PIF (India) & $=1.940$ \\
\hline & GIF (Australia) & $=0.564$ & ESJI (KZ) & $=8.716$ & IBI (India) & $=4.260$ \\
\hline & JIF & $=1.500$ & SJIF (Morocco & $=5.667$ & OAJI (USA) & $=0.350$ \\
\hline
\end{tabular}

множество конкурирующих стандартов, и еще больше находится разработке.

Сегодня существует несколько организаций, которые играют различные роли в разработке стандартов в здравоохранении. К таким относятся не только организации, непосредственно, занимающиеся разработкой стандартов, такие как HL7, DICOM [2], SNOMED и EHR, но также такие группы, как Integrating the Healthcare Enterprise (IHE), целью которых является не создание новых, а обеспечение скоординированного и последовательного использования различных уже доступных стандартов.

Для того, чтобы определить на какой стандарт следует обратить внимание, рассмотрим стандарты фирмы HL7, продуктами которой пользуются крупнейшие современные компании.

\section{Health Level 7 (HL7)}

В 1987 году была основана компания Health Level Seven International (HL7), которая в том же году выпустила свой первый стандарт, который носит одноименное название [3].

Стандарт HL7 предоставлял возможность единого представления медицинской документальной информации без разработки специальных программ и интерфейсов, т. е. стандартизировал обмен информацией, а не систем, которые передавали эти данные. Исходя их этого, появилось разнообразие методов применения данного стандарта в различных учреждениях здравоохранения.

\subsection{HL7 version 2}

Чуть позднее, в 1989 году была разработана первоначальная версия стандарта HL7 v2 специально для интеграции различных систем больниц, таких как административные и клинические системы.

Даже на сегодняшний день, HL7 v2 является принятым стандартом в больницах и местных сообществах и поддерживается большинством поставщиков медицинских информационных систем в Северной Америке [4]. Стандарт завоевал свой авторитет в том числе и благодаря тому, что был сформулирован довольно расплывчато, что давало медицинским организациям, имеющим, на тот момент довольно уникальные процессы в учреждениях, большую гибкость и легкость в реализации.

Тем не менее, HL7 v2 имеет несколько проблем:

1. Недостаточно масштабируется в более крупных многосторонних средах, таких как юрисдикционные информационные системы; например, в HL7 v2 отсутствует встроенная поддержка глобальных идентификаторов предприятий. Стандарт сильно зависит от локальной настройки благодаря использованию так называемых «Z-сегментов». Системные интерфейсы рассчитаны на $80 \%$, определяемые спецификациями HL7 и 20\%, адаптированные к локальной реализации [5].

2. Отсутствует формальная онтология, объединяющая обмены концепций между различными сообщениями и интерфейсами [6].

\subsection{HL7 version 3}

К 1998 году уже многие медицинские учреждения стали использовать стандарты, и HL7 v2 снискала все большую популярность и претерпела значительные изменения. Несмотря на это, основные проблемы стандарта новые версии не решали, и сообщество пришло к выводу о необходимости новой версии. Так, в 2005 году была создана первая версия HL7 v3, которая была не совместимой с HL7 v2. B первую очередь данное решение обусловлено тем, что была сделана попытка модифицировать типы данных и внедрить пользовательские роли. Кроме того, стандарту было необходимо качественное обновление для улучшения реализаций клинических интерфейсов [7].

Нововведением в HL7 v3 была информационная модель, называемая справочной информационной моделью или «RIM». RIM определил структуру на всех семантических и лексических элементах HL7 v3. RIM стремится структурировать всю информацию как «сущности» в «ролях», «участвующих» в «действиях» [8].

Несмотря на продуманность множества аспектов, стандарт имел несколько проблем:

1. Внедрение клинических моделей HL7 v3 требует сложных трансформаций модели в специфические для платформы модели, задачи, аналогичные задачам специального компилятора. HL7 такой инструмент не предоставляется.

2. Документация HL7 v3 утверждает [9], что стандарт предлагает семантическую интероперабельность, но из-за синтаксической сложности были примеры неправильных толкований, приводящие к последующим значительным переделкам.

\subsection{FHIR}

В январе 2011 года совет управляющих HL7 инициировал «новую целевую группу», чтобы изучить, как можно улучшить стандарты обмена сообщениями HL7. Это вдохновило независимую группу архитекторов HL7 начать обсуждение нового подхода к обмену информацией о здравоохранении, который они изначально называли «Ресурсы для здоровья - RFH» (впоследствии был переименован в «Fast Healthcare Interoperability Resources - FHIR») [10]. Этот новый подход был основан на принципах RESTful. 


\begin{tabular}{|c|c|c|c|c|c|c|}
\hline \multirow{4}{*}{ Impact Factor: } & ISRA (India) & $=3.117$ & SIS (USA) & $=0.912$ & ICV (Poland) & $=6.630$ \\
\hline & ISI (Dubai, UAE & $=0.829$ & РИНЦ (Russia & $=0.156$ & PIF (India) & $=1.940$ \\
\hline & GIF (Australia) & $=0.564$ & ESJI (KZ) & $=8.716$ & IBI (India) & $=4.260$ \\
\hline & JIF & $=1.500$ & SJIF (Morocce & $=5.667$ & OAJI (USA) & $=0.350$ \\
\hline
\end{tabular}

FHIR стремится определить ключевые субъекты, участвующие в обмене информацией о здравоохранении, в качестве ресурсов. Каждый ресурс представлял собой отдельный идентифицируемый объект.

Но это не избавило FHIR от проблем:

1. Стандарт считается достаточно новым, в связи с чем не очень распространен.

2. Как и стандарт HL7 v.3, FHIR поддерживает интероперабельность, но сложность состоит в том, что данное свойство должно поддерживаться семантикой, а стандарт это обеспечить не может.

\section{4 Выводы}

После рассмотрения стандартов HL7 была составлена сравнительная таблица (таблица 1), в которой подчеркиваются основные свойства каждого стандарта, что позволяет сравнить сильные и слабые стороны каждого из них. Несмотря на то, что стандарт FHIR привлекает большое внимание со стороны соответствующего сообщества [11] из-за его простоты (после архитектуры RESTful), нельзя недооценивать другие стандарты.

Тем не менее, очевидно, что в связи с быстрым ростом популярности, авторы FHIR пользуются опытом, накопленным в результате реализации своих предшественников, что значительно улучшает состояние информационного обмена между системами здравоохранения.

Таблица 1. Сравнение стандартов.

\begin{tabular}{|c|c|c|c|c|}
\hline Свойства & HL7 v2 & HL7 v3 & FHIR & OpenEHR \\
\hline Год создания & 1987 & 1997 & 2011 & 2003 \\
\hline Методология & Bottom up / ad hoc & Top-down, MDA & $\begin{array}{ll}\text { Iterative } & \text { and } \\
\text { incremental } & \\
\end{array}$ & Bottom up / ad hoc \\
\hline Парадигма & $\begin{array}{ll}\begin{array}{l}\text { Message, } \\
\text { and records }\end{array} & \text { Fields } \\
\end{array}$ & Message-Oriented & RESTful & Message-Oriented \\
\hline $\begin{array}{l}\text { Семантическая } \\
\text { онтология }\end{array}$ & Нет & Да & Да & Нет \\
\hline Поддержка & Поддерживается & Поддерживается & Поддерживается & Поддерживается \\
\hline $\begin{array}{l}\text { Степень } \\
\text { распространенности }\end{array}$ & Высокая & Очень низкая & Низкая & Очень низкая \\
\hline $\begin{array}{l}\text { Тип информационной } \\
\text { модели }\end{array}$ & ad hoc & constrained RIM & constrained RIM & ad hoc \\
\hline $\begin{array}{l}\text { Поддержка } \\
\text { международных } \\
\text { символов }\end{array}$ & Нет (ASCII) & Частично & Да (UTF8) & Частично \\
\hline
\end{tabular}

\section{References:}

1. Martselli, A. A. (2014). Latinskiy yazyk $i$ osnovy meditsinskoy terminologii / 3-e izd., ispr. (p.381).

2. Plotnikov, A. V., Prilutskiy, D. A., \& Selishchev, S. V. (1997). Standart DICOM v komp'yuternykh meditsinskikh tekhnologiyakh. Retrieved 2019, from https://mks.ru/library/article/1997/dicom.html

3. Namli, T., Aluc, G., \& Dogac, A. (2009). An Interoperability Test Framework for HL7-Based Systems. IEEE Transactions on Information
Technology In Biomedicine, 13(3), 389-399. doi: 10.1109/titb.2009.2016086

4. Otero, C., Luna, D., \& González Bernaldo de Quirós, F. (2018). Terminology Services: Standard Terminologies to Control Health Vocabulary. Yearbook of Medical Informatics, 27(01), 227-233. doi: 10.1055/s-0038-1641200

5. Dixon, B. (2016). Health information exchange: Navigating and Managing a Network of Health Information Systems. 


\begin{tabular}{|c|c|c|c|c|c|c|}
\hline \multirow{4}{*}{ Impact Factor: } & ISRA (India) & $=3.117$ & SIS (USA) & $=0.912$ & ICV (Poland) & $=6.630$ \\
\hline & ISI (Dubai, UAE & $=0.829$ & РИНЦ (Russia & $=0.156$ & PIF (India) & $=1.940$ \\
\hline & GIF (Australia) & $=0.564$ & ESJI (KZ) & $=8.716$ & IBI (India) & $=4.260$ \\
\hline & JIF & $=1.500$ & SJIF (Morocco & $=5.667$ & OAJI (USA) & $=0.350$ \\
\hline
\end{tabular}

6. Metke-Jimenez, A., Steel, J., Hansen, D., \& Lawley, M. (2018). Ontoserver: a syndicated terminology server. Journal of Biomedical Semantics, 9(1). doi: 10.1186/s13326-0180191-z

7. Luna, D., Otero, C., Gambarte, M., \& Frangella, J. (2018). Terminology Services: Standard Terminologies to Control Medical Vocabulary. "Words are Not What they Say but What they Mean". Ehealth - Making Health Care Smarter. doi: 10.5772/intechopen.75781

8. (n.d.). HL7 Reference Information Model. HL7. Retrieved 2019 , from http://www.hl7.org/implement/standards/rim.cf $\underline{\mathrm{m}}$
9. (n.d.). HL7 v3 (and ISO 21090). HL7. Retrieved 2019, from https://www.hl7.org/fhir/comparison-v3.html

10. (n.d.). FHIR Release 3 (STU). Terminology Service. FHIR. Retrieved 2019, from https://www.hl7.org/fhir/terminologyservice.html

11. Kopanitsa, G., \& Ivanov, A. (2018). Implementation of fast healthcare interoperability resources for an integration of laboratory and hospital information systems. Studies in Health Technology And Informatics, 247, 11-15. doi: 10.3233/978-1-61499-852-5-11 